\section{BIOINFORMATION \\ Discovery at the interface of physical and biological sciences}

BIOMEDICAL

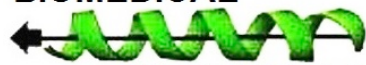

INFORMATICS
wWw.bioinformation.net

Volume 12(5)

\section{Hypothesis}

\title{
Insights from the analysis of predicted Rv0679c protein peptide from Mycobacterium tuberculosis with Toll like Receptors in host
}

\author{
Rupa Lavarti, Jayasree Ganugapati, Shirisa Ratcha, Lakshmana SS Rao, Krovvidi SR SivaSai*
}

Department of Biotechnology, Sreenidhi Institute of Science \& Technology, Ghatkesar, Hyderabad - 501301; Krovvidi S R SivaSai - Tel: (+91) 9949128880; E-mail:ksrsivasai@sreenidhi.edu.in; *Corresponding author,

Received May 16, 2016; Revised July 14, 2016; Accepted July 20, 2016; Published August 15, 2016

\begin{abstract}
:
Peptides of Rv0679c a membrane protein of the cell envelope (16.6 KDa) of Mycobacterium tuberculosis ( $M$. tb), inhibited entry of live bacilli into epithelial (A549) and macrophage (U937) cell lines in vitro, suggesting a possible role in invasion. Receptors associated with Rv0679c antigen entry into cell lines were not characterized. We are reporting that Rv0679c peptides could bind to Toll like receptors (TLRs), the principal class of pathogen recognition receptors on host cells (PRR) by docking studies. Peptide structures were predicted using PEP FOLD and docking of truncated peptides with TLR's was performed using Cluspro 2.0. Docked complexes were analyzed using Swiss-PDB Viewer. Nine peptides of Rv0679c protein assessed were able to bind to TLR2-1 and TLR 4-MD2; however the binding energy was better with TLR 4-MD2. Peptide $30985(-866.4 \mathrm{kcal} / \mathrm{mol})$ has better binding energy with TLR2-1, in contrast peptide 30982 showed a better binding energy to TLR 4-MD2 dimer with a score of $-1291.7 \mathrm{kcal} / \mathrm{mol}$. Interactive residue analysis revealed that GLU 173 and SER 454 of TLR 1; ARG 447 and ARG 486 of TLR2; ARG 264 of TLR 4 and SER 120, LYS 122 and GLU 92 of MD2 region are predominant residues interacting with peptides of Rv0679c protein. Our study suggests that predominant residues and receptors of TLR2 and TLR4 are important for Rv0679c protein binding, which could further lead to invasion of $M$. tb into the host cell.
\end{abstract}

Keywords: Mycobacterium tuberculosis, Rv0679c, toll like receptors, peptides, invasion.

\section{Background:}

Tuberculosis caused by Mycobacterium tuberculosis (M. $t b)$ is a major concern as new cases are diagnosed and many die because of the disease [1]. Emergence of acquired immune deficiency syndrome and development of multidrug-resistant mycobacteria has increased the risk of disease [2]. Hence, it is important to understand the host - pathogen interaction and find ways to stop the entry / invasion in host cells either in new individuals or individuals who are already diseased.

ISSN 0973-2063 (online) 0973-8894 (print)
Mycobacterium tuberculosis whole genome was sequenced [3], which led to deciphering the biology of the bacterium and predicted around 99 lipoproteins genes could be possible and Rv0679c gene was one of them [4]. Emphasis was on these envelope proteins and only few of them were experimentally characterized so far indicating their probable role in invasion [5]. However, Rv0679c gene / protein are not yet well characterized so far. Rv0679c protein (16.6 KDa) of M.tb was classified as an envelope protein consisting of 165 amino acids with a putative $\mathrm{N}$-terminal signal sequence and a consensus lipoprotein-processing motif using E. coli expression
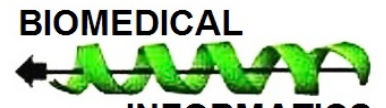

INFORMATICS 


\section{BIOINFORMATION \\ Discovery at the interface of physical and biological sciences}

\section{Open access}

system [3]. Study using globomycin treatment, Triton X-144 separation and mass spectrometry analysis revealed Rv0679c, a lipoprotein and exists as a tight complex with Lipoarbinomanan (LAM), one of the major components of cell envelope involved in pro-inflammatory and anti-inflammatory responses in $M$. $t b / M$. bovis BCG [6].

The functional role of either the Rv0679c gene or protein of $M . t b$ in pathogen-host interaction is not well known / characterized.
Recently, Nakajima C et al. 2013 [7] reported clinical significance of Rv0679c gene in demarcating the lineages of M.tb strains. They identified a unique SNP at position 426 where C/G only in Beijing genotype family of M.tb and developed a multiplex PCR assay for diagnostic purpose. We assessed the significance of Rv0679c gene of M. tb in Indian clinical isolates and reported that Rv0679c gene is highly conserved and SNP C426G was not observed in Indian clinical isolates indicating lineages are different except in one isolate which might be of Beijing origin [28].

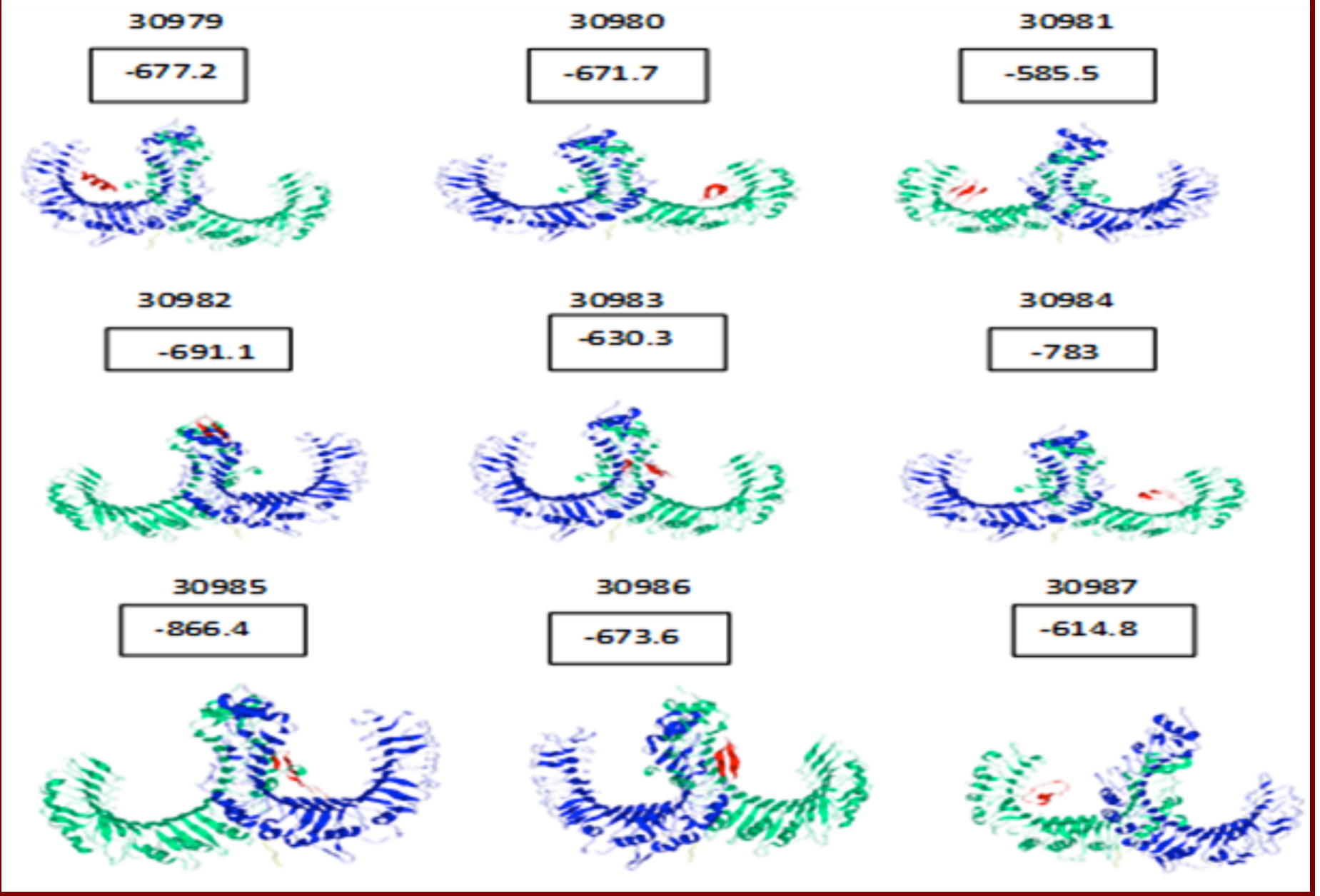

Figure 1: Structures of peptides of Rv0679c - TLR2-1 Docked complexes along with the binding energies is shown. Blue color of docked complex indicates TLR2, green indicates TLR1 and red indicates the peptides. Number in the parentheses indicates the order based on binding energy obtained in the peptide and docked complex. Negative values in the boxes indicate the binding energies in Kcal/mol while the numbers shown above the binding energies represents the peptide number.

BIOMEDICAL

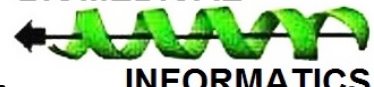




\section{BIOINFORMATION \\ Discovery at the interface of physical and biological sciences}

\section{Open access}

Cifuentes et al. [8] demonstrated for the first time that fragmented peptides of Rv0679c protein could selectively inhibit the entry of live $M$. $t b$ bacilli into epithelial (A549) and macrophage (U937) cell lines in vitro indicating a probable role of Rv0679c protein in invasion of the host. However, they have not assessed the receptors associated with entry/invasion or no reports are available in the literature regarding Rv0679c peptides/protein and its interaction with either TLRs or any other receptors on the host cells as crystallography structure of Rv0679c protein is not known. In view of this, we thought of assessing whether peptides of Rv0679c protein could bind to TLR receptors using Insilco approach.

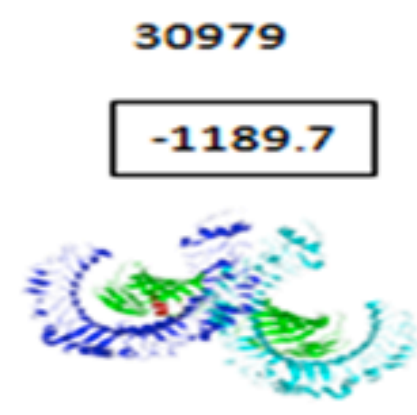

30982

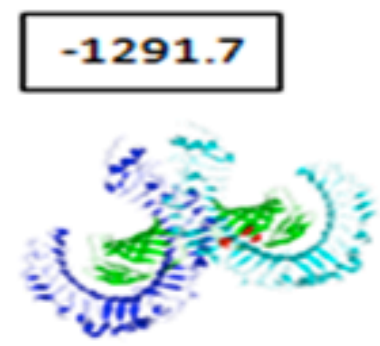

30985

\section{$-1053.6$}

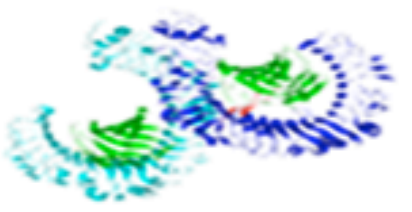

30980

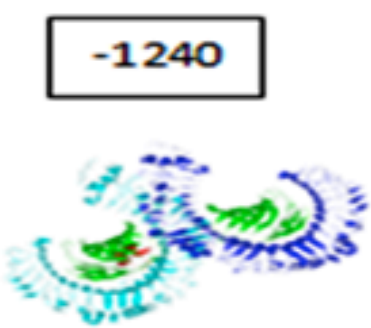

30983

1013.3

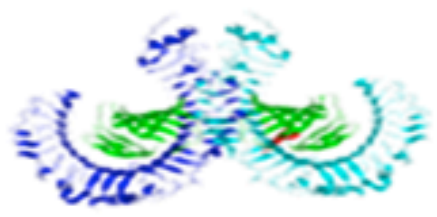

30986

$-1063$

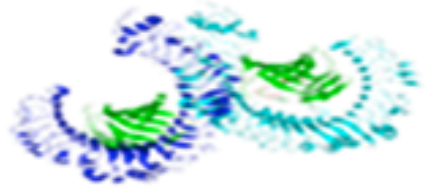

30981

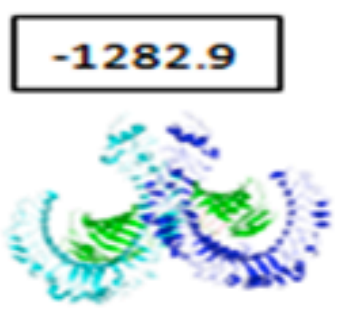

30984

$-1137.1$

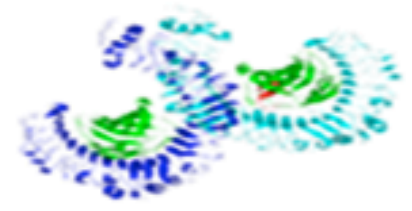

30987

$-1114.3$

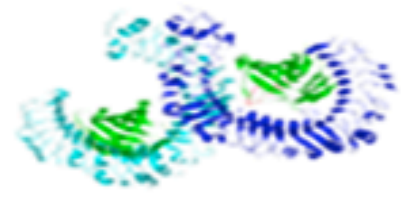

Figure 2: Structures of peptides of Rv0679c - TLR4-MD2 Docked complexes along with the binding energies. Blue color of docked complex indicates TLR4, green indicates MD2 complex and red indicates the peptides. Number in the parentheses indicates the order based on binding energy obtained in the peptide and docked complex. Negative values in the boxes indicate the binding energies in Kcal/mol while the numbers shown above the binding energies represents the peptide number. 


\section{BIOINFORMATION \\ Discovery at the interface of physical and biological sciences}

\section{Open access}

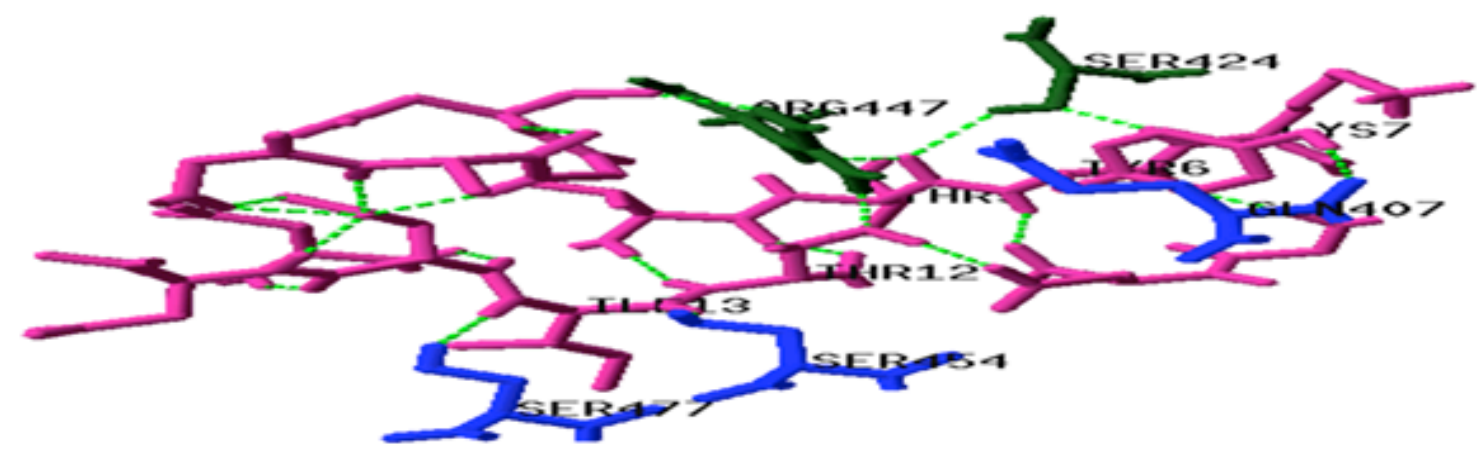

Peptide No

Interacting Residues of Rv0679c

Peptides-TLR 2-1 heterodimer.

30979

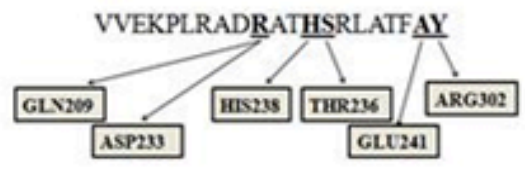

30986

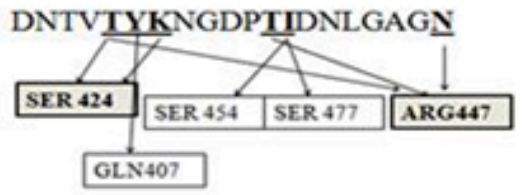

Interacting Residues of Rv0679c Peptides-TLR 2-1 heterodimer.
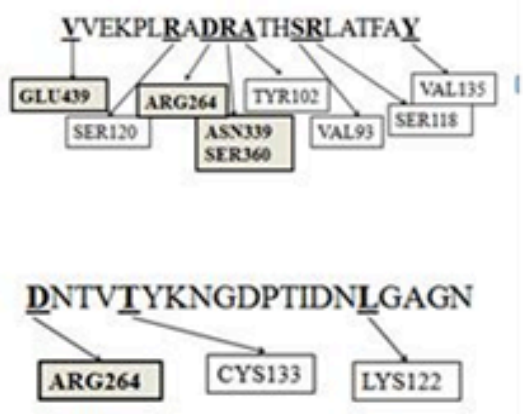

Figure 3: Representation of Structural view and identification of interactive residues of TLRs in docked complexes. A: Structural view of peptide 30986 and TLR2-1 docked complex; B: Interacting residues of TLR2-1 and TLR4-MD2 Dimer with peptides of Rv0679c antigen. Amino acids in bold are interactive residues of TLR-2 and TLR-4 and normal ones are interactive residues of TLR-1 and MD2 with peptides respectively. A representation of two peptides was depicted and the rest of the peptides were analyzed in a similar way. Residues underlined in peptides are interacting with TLR, which is depicted by arrows.

Toll-like receptors (TLRs) play a major role among the pattern recognition receptors and in infectious diseases, inflammatory diseases and cancer [9]. TLRs constitute a family of trans-membrane proteins expressed on many cells of the immune system and help in recognizing many microbial components like lipopeptides by TLR2 [10], lipopolysaccharide (LPS) by TLR-4 [11], flagellin by TLR-5 [12 \& 13] and bacterial CpG DNA by TLR-9 [14]. The expression of TLR-2 itself was induced by mycobacteria [15]. TLR-2 forms heterodimers with either TLR-1 or TLR-6 when they encounter triacylated or diacylated lipoproteins respectively [16]. TLR-2 being one of the main pattern recognition receptors for recognizing MTB, identification and characterization of mycobacterial TLR-2 ligands has gained importance.

Human TLR-4 detects lipopolysaccharide of gram-negative bacteria and activates the innate immune system [17]. TLR-4 consists of extracellular domain (608 residues), a single trans-membrane domain, and intracellular domain (187 residues) [18]. MD-2 (Myleoid differentiating factor), which lacks trans-membrane and intracellular regions, associates with the extracellular domain of TLR-4 and was believed to be the component of the TLR4-MD-2 complex that interacts with LPS [19]. The structure revealed that

\section{BIOMEDICAL}

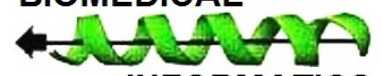

INFORMATICS 


\section{Open access}

MD-2 interacts with the N-terminal and central area of the concave surface of TLR-4.

Messenger RNA expression of TLRs 1-9 has been shown in human lungs, indicating a major site for TLR activity [20]. The identification of Rv0679c peptides ability to inhibit target cell invasion by $M . t b$ in cell lines suggests further studies related to host-cell receptors and their interactions for better understanding. Pulmonary TB being associated with lungs, we were interested in assessing the interaction of peptides of Rv0679c protein with Toll like receptors (TLRs) using Insilco approach.

\section{Methodology:}

Selection of Protein Sequence of Rv0679c and Structure Prediction of Peptides

The protein sequence of Rv0679c was retrieved from TubercuList database (http://tuberculist.epfl.ch). The sequence was truncated into 9 peptides $(20$ mer) spanning the entire length avoiding overlapping, identical to Cifuentes et al. [8] report and were assessed for docking studies with TLRs. The position of peptide and sequence being identical to Cifuentes et al. [8], we retained the same peptide number for comparative analysis as given below.

\begin{tabular}{lll}
\hline ID & Sequence & Position \\
\hline 30979 & VVEKPLRADRATHSRLATFAY & $1-20$ \\
30980 & LALAAAALPLAGCSSTANPPY & $21-40$ \\
30981 & AATTTPATATTTTATSGPTAY & $41-60$ \\
30982 & PTVTTGEST TASIQIGDMLY & $61-80$ \\
30983 & TYGSIGTTATLDCADGKSLN & $81-100$ \\
30984 & VAGSDNTLTVNGTCETVTVGY & $101-120$ \\
30985 & GANNKIAFDRIDERLV VVGLY & $121-140$ \\
30986 & DNTVTYKNGDPTIDNLGAGN & $141-160$ \\
30987 & YKNGDPTIDNLGAGNRINKE & $146-165$ \\
\hline
\end{tabular}

Structures of truncated peptides of Rv0679c were assessed by PEP FOLD SERVER (http://mobyle.rpbs.univ-paris-diderot.fr/cgibin/portal.py?form=PEP-FOLD\#forms::PEPFOLD).These structures were saved in PDB format and used for docking with TLR's.

\section{Retrieval of TLR structures}

TLR2-1 (PDB ID: 2Z7X) and TLR4-MD2 (PDB ID: 3FXI) structures were retrieved from Protein Data Bank (PDB) and were docked with peptides of Rv0679c antigen.

Docking Studies of Peptides of Rv0679c with TLR2-1 and TLR4MD2 heterodimers

Docking of truncated peptides with TLR's was performed using Cluspro 2.0 (http:/ / nrc.bu.edu/cluster) where in the peptides were considered as ligands while TLRs as receptor. Once the coordinate files of two protein structures are uploaded, through ClusPro's web interface, the docking algorithms evaluate billions of putative complexes, retaining a preset number with favorable surface complementarities. The obtained structures are then filtered, selecting those with good electrostatic and desolvation free energies for further clustering. The output generated was a short list of putative complexes ranked according to their clustering properties.

\section{Analysis of Interacting residues of the TLR-Peptide Complex}

The docked complexes of truncated peptides with TLR's, and the interacting residues were analyzed using Swiss-PdbViewer (SPDBV) (Guex,N) as given in Table 2. SPDBV provides a user friendly interface allowing analyzing several proteins at the same time. The software allows proteins to be superimposed in order to deduce structural alignments and compare their active sites or any other relevant parts. The interacting residues of TLRs with peptides fall within the TLR binding pockets which was further verified and confirmed using CASTP software.

\section{Results and Discussion:}

Prediction of 3D structures of truncated peptides of Rv0679c protein:

Structural analysis of truncated peptides revealed that peptide 30979, 30980 and 30987 showed $\alpha$ helices and random coil elements; peptides 30982, 30983, 30985 and 30986 showed $\beta$ sheets and random coil elements while peptides 30981 and 30984 didn't have either $\alpha$ helices or $\beta$ sheets and showed coils alone. These structures are saved in PDB format for docking studies with TLRs. These results are similar to and closely matched with circular dichroism studies carried out by Cifuentes et al. [8]. In our study we observed that the alpha helices peptides such as 30979, 30980 and 30987 in general showed lesser binding energies compared to Beta sheeted peptides to TLR2-1 and TLR4-MD2 complex. Beta-sheeted peptides 30985 and 30982 have highest / better binding affinity to TLR2-1 and TLR4-MD2 respectively.

Binding energy and mode of binding of truncated peptides of Rv0679c with TLR 2-1 and TLR 4 - MD2 heterodimer complexes TLR 2-1 and TLR-4 - MD2 heterodimer structures retrieved from RSCB PDB were docked with nine truncated peptide structures of Rv0679c antigen obtained using PEP FOLD server. The binding energy / scores obtained are depicted in Figures $1 \& 2$. The binding energy varied from - $585.5 \mathrm{Kcal} / \mathrm{mol}$ the lowest for peptide 30981 to $-866.4 \mathrm{Kcal} / \mathrm{mol}$ the highest for peptide 30985 of Rv0679c antigen interacting to TLR 2-1 complex (Figure 1). The binding energy of truncated peptides of Rv0679c to TLR 4-MD2 dimer varied from $-1013 \mathrm{Kcal} / \mathrm{mol}$ the lowest for peptide 30983 to -1291 Kcal / mol the highest for peptide- 30982 (Figure 2). Overall peptides of Rv0679c have better binding energies (20 - 100\%)

\section{BIOMEDICAL}

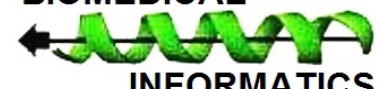


towards TLR-4 compared to TLR-2. Differential binding energies were observed between peptides of Rv0679c antigen to TLR-2 and TLR-4 dimers respectively. Peptide 30985 which has better binding energy towards TLR-2 has comparatively low affinity for TLR-4. Similarly, Peptide 30982 has better binding energy to TLR-4 compared to TLR-2. Noteworthy is the peptide 30982 which was showing high binding affinity to TLR-4 in our study, had minimal affect on inhibition of invasion of M.tb into cell lines in Cifuentes et al. [8] study, suggesting TLR-4 may not be involved in invasion.

We compared inhibition of cell invasion studies in vitro into cell lines by Cifuentes et al. [8] with our own findings of peptide docking studies (binding energy) to TLRs, as the peptides in both studies are identical. Surprisingly, peptides 30979 and 30987 which showed strong inhibition in their invasion studies were binding to TLR2-1 heterodimer and TLR-4 MD2 dimer with lower binding ability. Localization analysis of TLR-peptide ligand interaction revealed that peptide 30979 binds to exclusively to TLR-2 domain and peptide 30987 to TLR-1domain in the TLR-2-1 heterodimer. The interactive residue analysis of TLR and peptides indicated that the TLR-2 and TLR-1 residues associated with respective peptides are unique and majority of them are not repeated with other peptides. Taken together we speculate that pretreatment of cell lines with the peptides 30979 and 30987 selectively binding to TLR-2 and TLR-1 domains respectively might have led to inhibition of the formation of hetero dimerization of TLR2-1 which is important for entry of pathogen. TLR-2 in association with TLR-1 or TLR-6 plays an important role in the innate immune response by recognizing microbial lipoproteins and lipopeptides [21]. Jin MS et al [16] showed that tri-acylated lipopeptide Pam3CSK4 and di-acylated lipopeptide Pam2CSK4 even though bind to TLR-2, a proper heterodimer formation was induced only with Pam3CSK4 but not with Pam2CSK4.

Peptide 30985 has better binding ability to TLR-2-1 heterodimer out of the nine peptides assessed compared to TLR4-MD2 dimer where the peptide stand at eight position, indicating its preference towards TLR-2. Noteworthy is the peptide 30985 which was binding better energy to TLR-2 in our study correlates well with the findings of Cifuentes et al. [8] who showed that at low concentration it is efficient in inhibition compared to high concentrations where the inhibition is maximum and leading to cytotoxicity on A549 and U 937 cell lines. In other words this peptide failed to respond in a dose dependent manner in their study. Peptide 30982 has better binding affinity to TLR-4 in our study. The same peptide could not inhibit the invasion/entry of $M$. $t b$ into the peptide pretreated cell lines indicating lack of inhibition. Hence this peptide was used as a negative control in their study which turns out to be a better TLR-4 binder suggests that TLR-4 might not be the receptor involved in invasion.

\section{Assessment of Interactive residues of TLRs with Peptides of Rv0679c antigen in docked complexes:}

Peptides of Rv0679c antigen bound to TLR2-1 and TLR4-MD-2 regions. A representative illustration showing the interactive residues of TLR 2-1 and the peptide 30986 is given in Figure 3A. The interacting amino acid residues of TLR 2-1 hetero-dimer and TLR 4-MD2 dimer with two peptides are represented in Figure 3B, even though all peptides are assessed in the same way and the results are summarized in the text.

The peptide 30980, 30981, 30984 and 30987 of Rv0679c antigen bound to TLR-1 region and peptide 30979 to TLR-2 region exclusively, compared to remaining four peptides which are bound to both TLR-1 and TLR-2 of TLR 2-1 hetero-dimer. Out of the peptides exclusively binding to TLR-1 region the predominant common residue in TLR-1 was GLU-173. The common interactive residues in TLR-2 were ARG 447 and ARG 486 and in TLR-1 was SER-454 for the peptides which are bound to both TLR 2-1 heterodimer. Interestingly, Peptide 30979 and 30987 which was binding exclusively to TLR-2 and TLR1 in a TLR2-1 hetero-dimer respectively has different/unique residues which predominantly do not match with other peptides. Note worthy is that these two peptides showed maximum inhibition of entry of $M$. $t b$ into human A549 and U937 cells lines in Cifuentes et al. [8] study.

Except Peptide 30981, which was exclusively bound to MD2, region the rest of the peptides of Rv0679c were bound to TLR-4 and MD2 region of TLR 4-MD2 dimer. However, majority of interactive residues fall into MD 2 region except in peptide 30979, 30982 and 30985 where TLR- 4 was predominant. In seven of the 9 peptides of Rv0679c assessed ARG 264 was found to crucial residue of TLR-4 interacting with peptides. Out of the MD2 region SER 120, LYS 122 and GLU 92 were found to predominant interactive residues with peptides of Rv0679c antigen. We looked into whether the predominant interacting residues identified in our study are conserved across the TLRs assessed because LRR modules of TLR consist of conserved "xLxxLxxLxLxxNxLxxLPxxxFx" motifs [22]. We could not find any commonality/similarity between predominant residues identified in our study across the TLRs assessed. This might be because LRR modules of TLR 1, 2, 4 and 6 deviate from their conformation and length and also lack an asparagine ladder leading to unusual structural distortion, because asparagine helps in stabilization of horseshoe like structure [17, 22]. Structural conformational variations of above TLR receptors could explain the ability to bind with diverse ligands.
BIOMEDICAL

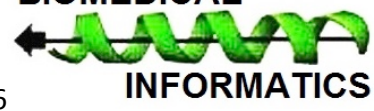




\section{Open access}

Even though the Rv0679c protein is a hypothetical / putative membrane lipoprotein, at this point we have not assessed the significance of lipid component in terms of interaction, signaling and other related functions. Different groups have showed that live bacilli, lysate or proteins of M.tb were able to inhibit MHC class-II expression and antigen processing [23], of the host and thus evade host immunity. Lipoproteins of M.tb such as LprA [24] and LprG [25] were shown to activate these cells via both TLR-2 and TLR-4, and selectively inhibit MHC expression and generalized antigen processing to multiple antigenic epitopes which was dependent on TLR-2 and independent of TLR-4 [26]. Studies by Abel et al, found an increased susceptibility to M.tb infection of TLR-4 mutant mice in terms of a reduced survival and an impaired mycobacterial clearance [27]. TLR4 plays a protective role in host defense against pulmonary tuberculosis in vivo, as reflected by an increased mortality and mycobacterial load in the lungs of mice with a non-functional TLR-4. Overall, based on our results and findings of Cifuentes et al. [8] we conclude, Rv0679c antigen prefer TLR-2 receptor over TLR-4 for invasion of M.tb pathogen into the host cell. Within the Rv0679c protein, peptide 30979 and 30987 are more relevant in terms of selective residue interaction and good binding energy with TLR-2.

\section{Conclusion:}

Peptides of Rv0679c protein bind to TLR-2 and TLR-4 however peptides 30979, 30987 and 30982, 30985 preferred binding to TLR2 and TLR4 respectively. One would assume route of entry or invasion of the pathogen into the host depends on interacting residues at that given point of time which could determine the outcome. Studies based on invasion of $M$. tb using it's envelop antigens into the host using TLRs [24, 25], Cifuentes et al. [8] invasion studies using Rv0679c peptides and our own findings we speculate that Rv0679c antigen prefers TLR-2 receptor over TLR-4 for invasion of $M$. $t b$ pathogen into the host cell. Within the Rv0679c protein, peptides 30979 and 30987 are being preferably_associated with TLR2; interception of these interactions by intervening strategies could be beneficial in a disease scenario.

\section{Acknowledgements:}

We express our in-depth thanks to Indian Council of Medical Research (ICMR) New Delhi, India for Senior Research Fellowship (SRF) assistance to Lavarti Rupa (File No: 80/747/2012-ECD-I). The group would like to express their appreciation to the management and administration of Sreenidhi Institute of Science and Technology and TEQIP-II program, World Bank Scheme for their support of the study.

\section{References:}

[1] World Health Organization Global Tuberculosis Report 2014 [PMID: 25539957]

[2] Kaufmann SH. \& McMichael AJ, Nature Medicine 2005 11(4): S33 [PMID: 15812488]

[3] Cole S. T et al. Nature 1998 393: 537 [PMID: 9634230]

[4] Andersen $P$ et al. Infect Immun. 2000 356: 1099 [PMID:11009160]

[5] Sutcliffe IC et al. FEMS Microbiol Rev. 2004 28(5): 645 [PMID:15539077]

[6] Matsuba T et al. Arch Microbiol. (2007) 187: 297 [PMID: 17252234]

[7] Chie Nakajima et al. J Clin Microbiol. 2013 51(7): 2025 [PMC ID:3697683]

[8] Cifuentes DP et al. BMC Microbiology. 2010 (4)10: 109 [PMID: 20388213]

[9] O'Neill LA et al. Pharmacol. Rev. 2009 61(2): 177. [PMID: 19474110]

[10] Akira S \& Takeda K Nat. Rev. Immunol 2004 4: 499 [PMID: 15229469]

[11] Tapping RI et al. J Immunol. 2000 165: 5780 [PMID: 11067937]

[12] Hayashi F et al. Nature. 2001 410: 1099 [PMID: 11323673]

[13] Gewirtz AT et al. J. Immuno. 2001 167: 1882 [PMID: 11489966]

[14] Hemmi H et al. Nature. 2000 408: 740 [PMID: 11130078]

[15] Wang T et al. J Immuno. 2000 165: 6308 [PMID: 11086067]

[16] Jin MS et al. Cell. 2007 130(6): 1071 [PMID:17889651]

[17] Kim HM et al. Cell. 2007 130(5): 906 [PMID: 17803912]

[18] Medzhitov et al. Nature. 1997 388: 394 [PMID: 9237759]

[19] Shimazu, R et al. J. Exp. Med. 1999 189: 1777 [PMID: 10359581]

[20] Zarember KA \& Godowski PJ. J Immunol. 2002 168(2): 554 [ PMID:11777946]

[21] Takeuchi O et al. J Immunol. 2002 169(1): 10 [PMID:12077222]

[22] Balachandran M et al. Frontiers in Physiology. 2011 2(41): 1

[23] Pecora ND et al. J Immunol. 2006 177(1): 422 [PMID:16785538]

[24] Jo EK et al. Cell Microbiol. 2007 9(5): 1087 [PMID: 17359235]

[25] Gehring AJ et al. J Immunol. 2004 173(4): 2660 [PMID:15294983]

[26] Noss EH et al. J Immunol 2001 167: 910 [PMID: 11441098]

[27] Abel B et al. J. Immunol. 2002 169: 3155 [PMID:12218133]

[28] Lavarti Rupa et al. IJMM, 2016 34(4): 1-5

Edited by P Kangueane Citation: Lavarti et al. Bioinformation 2016 12(5) 293-299. License statement: This is an Open Access article which permits unrestricted use, distribution, and reproduction in any medium, provided the original work is properly credited. This is distributed under the terms of the Creative Commons Attribution License.
ISSN 0973-2063 (online) 0973-8894 (print)

\section{BIOMEDICAL}

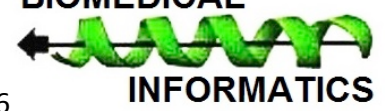

INFORMATICS

Bioinformation 12(5): 293-299 (2016)

(C)2016 